\title{
高活性单分散磺化聚苯乙烯多孔微球用于生物柴油制备研究
}

\author{
罗建新 ${ }^{a} \quad$ 颜文海 $^{a}$ 马青 ${ }^{a}$ 张春燕 $*, a$ \\ 方怡权 ${ }^{b}$ 张栩诚 ${ }^{b}$ 汪长春 ${ }^{*}, b$ \\ ( ${ }^{a}$ 湖南工学院材料与化学工程学院 衡阳 421002) \\ $\left({ }^{b}\right.$ 复旦大学高分子科学系 聚合物分子工程国家重点实验室 上海 200433)
}

\begin{abstract}
摘要 采用回流-沉淀聚合法高效制备了单分散交联多孔聚苯乙烯微球, 再在温和的条件下对其进行磺化, 制备了磺 化聚苯乙烯多孔微球. 以调控磺化度和酸密度为目标, 重点考察了溶剂用量、溶胀时间、氯磺酸用量、磺化温度和磺 化时间等影响因素. 用 $5 \mathrm{~mL} \mathrm{CCl}_{4}$ 溶胀 $0.5 \mathrm{~g}$ 交联聚苯乙烯微球, 然后加入 $0.3 \mathrm{~mL}$ 氯磺酸, 在 $50{ }^{\circ} \mathrm{C}$ 磺化 $75 \mathrm{~min}$, 磺化度 和酸密度分别可以达到 $85.1 \%$ 和 $2.611 \mathrm{mmol} \cdot \mathrm{g}^{-1}$. 该磺化聚苯乙烯微球在催化油酸和甲醇酯化合成生物柴油中表现出 很高的催化活性，远高于酸性离子交换树脂 Ameberlyst-15，接近于均相的浓硫酸体系; 且循环使用 3 次后，催化活性仍 可保持初始活性的 $92 \%$. 提供了一种简单、绿色和可控的方法, 制备了酸密度可控、稳定性好的单分散磺化聚苯乙烯 微球, 在工业制备生物柴油领域具有较好的前景.
\end{abstract}

关键词＼cjkstart回流-沉淀聚合; 多孔聚苯乙烯微球; 磺化; 生物柴油

\section{Study on High Activity Monodispersed Sulfonated Porous Polystyrene Microspheres for Preparation of Biodiesel}

\author{
Luo, Jianxin $^{a} \quad$ Yan, Wenhai $^{a} \quad$ Ma, Qing ${ }^{a} \quad$ Zhang, Chunyan ${ }^{*, a}$ \\ Fang, Yiquan $^{b}$ Zhang, Xucheng ${ }^{b}$ Wang, Changchun ${ }^{*, b}$ \\ ( ${ }^{a}$ Department of Materials and Chemical Engineering, Hunan Institute of Technology, Hengyang 421002) \\ $\left({ }^{b}\right.$ State Key Laboratory of Molecular Engineering of Polymers, Department of Macromolecular Science, Fudan \\ University, Shanghai 200433)
}

\begin{abstract}
Sulfonated porous polystyrene microspheres as heterogeneous catalyst for preparation of biodiesel were prepared by a facile two-step synthesis process in this work. Firstly, monodisperse porous cross-linked polystyrene microspheres were prepared by reflux-precipitation polymerization, in which different ratio of styrene (St) and divinylbenzene (DVB) were used as monomer and cross-linker, respectively. Then the obtained polystyrene microspheres were sulfonated by chlorosulfonic acid. The structure and composition of the related polystyrene microspheres were characterized by FT-IR, X-ray photoelectron spectroscopy, elemental analysis, thermogravimetric analysis, Brunauer-Emmett-Teller (BET) surface area analysis, transmission electron microscope, particle size and zeta potential analysis. In order to adjust the sulfonated degree and acid density, the reaction parameters such as solvent dosage, swelling time, amount of chlorinated sulfonic acid, sulfonated temperature and sulfonated time were investigated carefully. The optimum conditions for the sulfonated reaction are as follows: $0.5 \mathrm{~g}$ of cross-linked polystyrene microspheres was swollen with $5 \mathrm{~mL}$ of $\mathrm{CCl}_{4}$, and then sulfonated with $0.3 \mathrm{~mL}$ of chlorosulfonic acid at $50{ }^{\circ} \mathrm{C}$ for $75 \mathrm{~min}$. In addition, the effect of crosslinking degree on the specific surface area and acid density of the sulfonated polystyrene microspheres were also studied. The as-prepared sulfonated polystyrene microspheres exhibited high acid density $\left(2.611 \mathrm{mmol} \bullet \mathrm{g}^{-1}\right)$, good thermal stability (up to about $200{ }^{\circ} \mathrm{C}$ ) and appropriate specific surface area. The sulfonated porous polystyrene microspheres as catalyst were applied in the esterification reaction of oleic acid and methanol. Our experimental results showed that the functional particles possessed very high catalytic activity, which was much higher than polystyrene ion exchange resin (Ameberlyst-15) and close to concentrated sulfuric acid. The catalytic activity still maintained $92 \%$ of the initial activity after 3 runs of recycling use, and the shedding of sulfonic acid groups was almost negligible. This paper demonstrates a simple, green and controllable strategy to develop sulfonated porous polystyrene microspheres with high catalytic activity and good durability for preparation of biodiesel.

Keywords reflux-precipitation polymerization; polystyrene microsphere; sulfonated reaction; biodiesel
\end{abstract}

\footnotetext{
* E-mail: chinachunyan@126.com; ccwang@fudan.edu.cn

Received August 15, 2018; published October 8, 2018.

Supporting information for this article is available free of charge via the Internet at http://sioc-journal.cn.

Project supported by the National Natural Science Foundation of China (No. 21802039), the National College Students' Innovation and Entrepreneurship Training Program Project (Jiao Gao Si No. [2017]40), Hunan Province College Students' Research Learning and Innovative Experiment Project (Xiang Jiao Tong Nos. [2017]205, [2018]255) and the Construct Program of the Key Discipline in Hunan Province (Xiang Jiao Fa No. [2011]76).

项目受国家自然科学基金(No. 21802039)、国家级大学生创新创业训练计划(教高司函[2017]40 号)、湖南省大学生研究性学习和创新性实验计划(湘教 通[2017]205 号, 湘教通[2018]255 号)和湖南省重点建设学科(湘教发[2011]76 号)资助.
} 


\section{1 引言}

在化石燃料储量逐步下降、环境问题日益严峻的背 景下, 生物燃料受到各国政府的高度重视. 其中, 生物 柴油的燃烧特性与柴油相似, 可以直接用于现有的柴油 机; 而且, 生物柴油中高的氧含量能够提高燃烧性能并 降低 CO、积碳和碳氧化合物的释放; 与化石燃料相比, 生物柴油几乎不含硫和芳香类物质, 可被生物降解且无 毒, 是一种部分替代化石柴油的绿色燃料 ${ }^{[1 \sim 3]}$.

液体强酸 $\left(\mathrm{H}_{2} \mathrm{SO}_{4}\right)$ 催化制备生物柴油的效率较高, 但存在催化剂回收利用困难、产物后处理繁琐、设备腐 蚀严重和废液排放较多等诸多问题 ${ }^{[4,5]}$. 采用介孔硅固 体酸和碳基固体酸等非均相酸催化剂可有效克服上述 问题 ${ }^{[4]}$. 但是, 介孔硅制备过程较复杂, 成本高, 能耗 大、酸稳定性不佳, 且介孔硅的疏水性差; 碳基固体酸 制备需经过高温碳化和磺化等过程, 也存在能耗大及污 染大等问题, 且其酸性位点容易脱落, 稳定性一般. 聚 合物具有较好的耐酸碱性和疏水性, 制备简单且易修 饰, 共价键连接的酸性位点易调控, 稳定性高 ${ }^{[9 \sim 11]}$. 但 是, 聚合物的酸性位点易被包裏, 不利于催化反应. Liu 等 ${ }^{[12]}$ 将二乙烯基苯和对苯乙烯磺酸钠在溶剂热条件下 共聚制备得到疏水性介孔聚合物基固体酸催化剂; 具有 丰富的介孔、比表面积较大但是酸密度较低 $(0.26 \sim 1.86$ $\left.\mathrm{mmol} \cdot \mathrm{g}^{-1}\right)$ 等特点. Zhang 等 ${ }^{[13]}$ 先通过乳液聚合制备聚苯 乙烯模板微球, 然后制备了 PS@介孔硅蛋黄-蛋壳型纳 米微球, 最后用氯磺酸磺化制备磺化 PS@介孔硅双壳 型纳米微球; 在中空硅的纳米空间里, 通过磺酸基的氢 键自组装提高酸密度和相邻酸性位点间的协同作用, 从 而提高其酸强度. 虽然该催化剂的催化活性较高, 但是 其制备过程较复杂. 因此, 采用简单、绿色和可控的方 法, 制备酸密度高、稳定性好的新型聚合物基固体酸催 化剂具有重要的现实意义.

多孔聚合物微球的比表面积较大, 表面易修饰酸性 基团, 且表面修饰的酸性位点位阻小, 在催化制备生物 柴油等方面潜力很大. 单分散聚合物微球的制备常采用 乳液聚合、微乳液聚合、细乳液聚合及分散聚合, 在聚 合过程中往往需要添加稳定剂或乳化剂, 对微球的分离 纯化造成了困难，限制了其在催化方面的潜在应用. 我 们课题组 ${ }^{[14]}$ 新近发展的回流-沉淀聚合技术非常适合该 类微球的制备, 获得的微球表面清洁、单分散性好、操 作简单、耗时短. 因此, 本工作采用回流-沉淀聚合法制 备了交联聚苯乙烯多孔微球, 然后对其进行磺化, 制备 磺化聚苯乙烯多孔微球, 考察了相关影响因素, 并用于 催化油酸和甲醇酯化制备生物柴油研究.

\section{2 结果与讨论}

\section{1 磺化聚苯乙烯微球的制备与表征}

以苯乙烯 $(\mathrm{St})$ 为单体、二乙烯基苯(DVB)为交联剂、
偶氮二异丁腈(AIBN)为引发剂, 乙腈为溶剂, 采用回 流-沉淀聚合制备交联聚苯乙烯微球 ${ }^{[14]}$. 改变 St/DVB 的 配比, 制备得到四种聚合物微球, 如表 1 所示. 可溶性 单体聚合形成寡聚物，当其分子量达到一定值即从溶剂 中析出形成核，核表面的双键继续捕捉溶液中的单体或 寡聚物进行增长, 形成稳定粒子. 交联单体的用量越大, 粒子表面的双键就越多, 粒子表面的双键相互聚合可能 性较大, 导致聚合物微球聚集, 粒径增加且分布变宽. 如果交联单体用量较低, 则聚合物微球的交联度较低, 微球易溶胀、稳定性较差. 综合考虑, St/DVB 的质量比 为 1 时，回流-沉淀聚合制备的聚合物微球(PM-1)粒径 较均匀、稳定性较好.

表 1 交联聚苯乙烯微球的结构和性能参数

Table 1 Structure and performance parameters of cross-linked polystyrene microspheres

\begin{tabular}{ccccccc}
\hline Microsphere & St $/ \mathrm{mg}$ & DVB/mg & Cons. $/ \%$ & $d / \mu \mathrm{m}$ & PDI & $\zeta / \mathrm{mV}$ \\
\hline PM-1 & 450 & 450 & 63.6 & 1.28 & 0.081 & -5.5 \\
PM-2 & 300 & 600 & 63.7 & 1.46 & 0.102 & -4.8 \\
PM-3 & 150 & 750 & 60.9 & 1.53 & 0.105 & -4.6 \\
PM-4 & 0 & 900 & 59.9 & 1.66 & 0.133 & -4.1 \\
\hline
\end{tabular}

选用上述所制备的聚苯乙烯微球 PM-1 为原料, 以 $\mathrm{CCl}_{4}$ 为溶剂, 用氯磺酸磺化制备磺化聚苯乙烯微球, 用 红外光谱和 $X$ 射线光电子能谱对其进行表征(图 1). 与 PM-1 相比, 磺化聚苯乙烯微球 PM-1S 在 $3420 \mathrm{~cm}^{-1}$ 处 的羟基峰明显增强, 在 $1637 \mathrm{~cm}^{-1}$ 处的苯环骨架振动峰 增强并变宽, 在 $1176 \mathrm{~cm}^{-1}$ 处出现磺酸基的 $\mathrm{S}-\mathrm{O}$ 反对称 伸缩振动峰 ${ }^{[8,15]}$. 由于苯环 $\mathrm{C}-\mathrm{H}$ 面内弯曲振动受 $\mathrm{S}-\mathrm{O}$ 对称伸缩振动的影响, PM-1S 在 $1067 \mathrm{~cm}^{-1}$ 处的吸收分 裂为双重峰 $\left(1093,1043 \mathrm{~cm}^{-1}\right)$. 并且, 相比于 PM-1, PM-1S 的 X 射线光电子能谱在 169 和 $535 \mathrm{eV}$ 处分别出 现了 $S(2 p)$ 和 $O(1 s)$ 的特征峰(图 $1 b)$. 这些结果均表明磺 酸基已被成功引入苯环. 由图 2 可见, 聚苯乙烯微球 PM-1 和磺化聚苯乙烯微球 PM-1S 均显示完美的球形结 构, 且粒径相对均一; 说明磺化未改变聚苯乙烯微球的 形貌. 比较发现, 磺化后微球的流体力学粒径 (d)增大、 Zeta 电位(ら)下降(表 1 和表 2 ), 说明磺化后微球表面负电 荷密度增加, 也证明了磺酸基已被成功引入微球表面.

采用单因素实验，考察溶胀时间、磺化时间、溶剂 用量、磺化温度、氯磺酸用量等因素对聚苯乙烯微球磺 化反应的影响. 溶胀时间 $\left(t_{1}\right)$ 由 $0 \mathrm{~h}$ 延长至 $5.5 \mathrm{~h}$, 微球的 磺化度 $\left(D_{\mathrm{S}}\right)$ 变化较小, 均在 $83.1 \% \sim 85.7 \%$ 范围内, 酸密 度 $\left(\left[\mathrm{H}^{+}\right]\right)$变化也较小. 从效率和节能的角度考虑, 溶胀 时间选择为 $0 \mathrm{~h}$. 随着磺化时间 $\left(t_{2}\right)$ 的延长, $D_{\mathrm{S}}$ 也逐渐增 加, 当磺化 $75 \mathrm{~min}$ 后, 其 $D_{\mathrm{S}}$ 不再增加. 这是因为随着时 间的延长, 氯磺酸逐渐浸入微球内部, $D_{\mathrm{S}}$ 得到提高; 磺 化超过 $75 \mathrm{~min}$ 后, 氯磺酸浸入微球内部已经达到平衡, $D_{\mathrm{S}}$ 也不再变化; $\zeta$ 和 $\left[\mathrm{H}^{+}\right]$与 $D_{\mathrm{S}}$ 的变化规律类似. 由此可 见, 磺化 $75 \mathrm{~min}$ 较佳. 当溶剂的量少于 $5 \mathrm{~mL}$ 时, 交联聚 

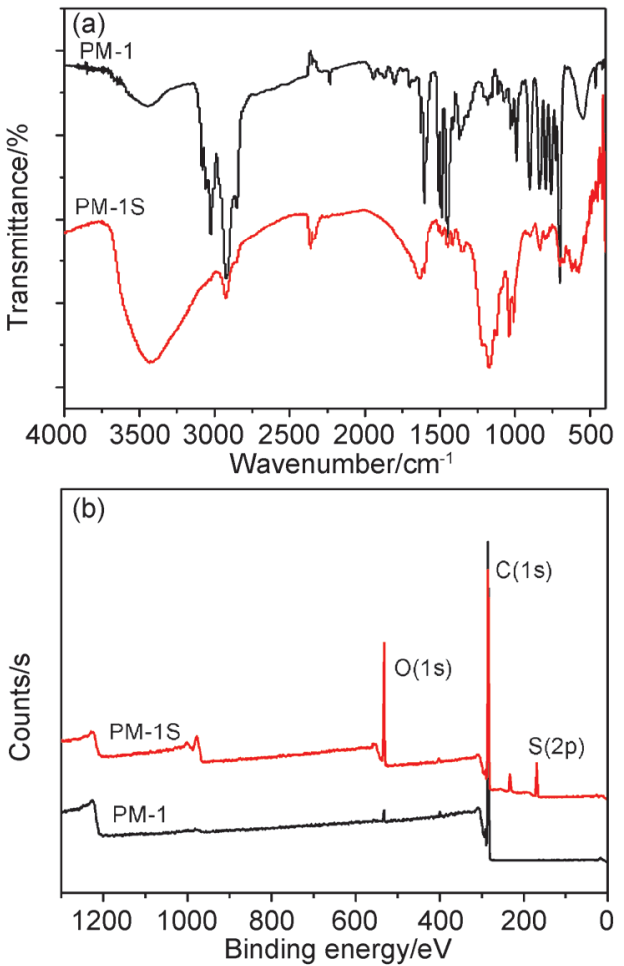

图 1 聚苯乙烯微球(PM-1)和磺化聚苯乙烯微球(PM-1S)的(a)红外光 谱和(b)X 射线光电子能谱

Figure 1 FT-IR (a) and XPS (b) of polystyrene microspheres (PM-1) and sulfonated polystyrene microspheres (PM-1S)

苯乙烯微球难以充分溶胀, 导致氯磺酸无法浸入微球 中, 从而 $\zeta 、 D_{\mathrm{S}}$ 和 $\left[\mathrm{H}^{+}\right]$下降; 而溶剂过量时, 聚苯乙烯微 球的浓度降低, 反应速度下降, 因而 $\zeta 、 D_{\mathrm{S}}$ 和 $\left[\mathrm{H}^{+}\right]$也下

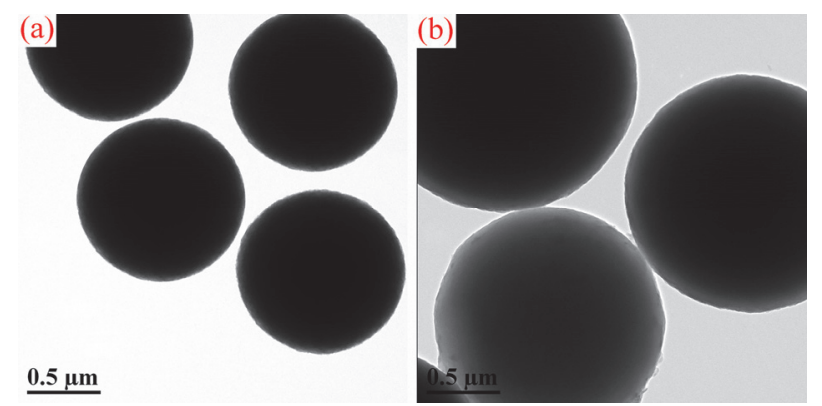

图 2 (a)聚苯乙烯微球 PM-1 和(b)磺化聚苯乙烯微球 PM-1S 的 TEM Figure 2 TEM of (a) polystyrene microspheres PM-1 and (b) sulfonated polystyrene microspheres PM-1S

降. 因此, 当溶剂用量为 $5 \mathrm{~mL}$ 时, 磺化效果最佳. 随着 磺化温度的提高，其磺化度也得到提高，这是由于升高 温度，分子链易运动，氯磺酸易浸入微球内部，进而 $D_{\mathrm{S}}$ 提高, $d, \zeta$ 和 $\left[\mathrm{H}^{+}\right]$也得到了提高. 䇻选出较佳磺化温度为 $50{ }^{\circ} \mathrm{C}$. 随着氯磺酸用量的增加, 磺化聚苯乙烯微球的 粒径 $d$ 增加, $\zeta, D_{\mathrm{S}}$ 和 $\left[\mathrm{H}^{+}\right]$先增加, 而后基本不变. 当氯磺 酸用量为 $0.3 \mathrm{~mL}$ 时, $\zeta, D_{\mathrm{S}}$ 和 $\left[\mathrm{H}^{+}\right]$相对较高, 酸催化活性 较高. 综合考虑, 聚苯乙烯微球的最佳磺化条件是：聚 苯乙烯微球 $0.5 \mathrm{~g}$, 溶剂 $\mathrm{CC}_{4}$ 用量为 $5 \mathrm{~mL}$, 溶胀时间为 0 $\mathrm{h}$, 氯磺酸用量为 $0.3 \mathrm{~mL}$, 磺化温度为 $50{ }^{\circ} \mathrm{C}$, 磺化 75 min 得到磺化聚苯乙烯微球的磺化度和酸密度最高, 高 于大多数碳基固体酸和聚合物基固体酸 ${ }^{16 ~ 19]}$.

在上述最佳磺化条件下, 分别将聚苯乙烯微球 PM-1, PM-2, PM-3 和 PM-4 磺化, 制备得到磺化聚苯乙 烯微球 PM-1S, PM-2S, PM-3S 和 PM-4S. 由图 3a 可见,

表 2 磺化聚苯乙烯微球的制备条件和性能参数

Table 2 Preparation conditions and properties of the sulfonated polystyrene microspheres

\begin{tabular}{cccccccccc}
\hline Sample & $t_{1} / \mathrm{h}$ & $t_{2} / \mathrm{min}$ & $\mathrm{CCl}_{4} / \mathrm{mL}$ & $T / \mathrm{C}$ & $\mathrm{HSO}_{3} \mathrm{Cl} / \mathrm{mL}$ & $d / \mu \mathrm{m}$ & $\zeta / \mathrm{eV}$ & $D_{\mathrm{S}} / \%$ & {$\left[\mathrm{H}^{+}\right] /\left(\mathrm{mmol} \bullet \mathrm{g}{ }^{-1}\right)$} \\
\hline PM-1S1 & 0 & 60 & 5 & 50 & 0.3 & 1.87 & -27.3 & 84.9 & 2.542 \\
PM-1S2 & 1 & 60 & 5 & 50 & 0.3 & 2.06 & -26.6 & 83.7 & 2.423 \\
PM-1S3 & 2 & 60 & 5 & 50 & 0.3 & 2.15 & -26.9 & 83.1 & 2.395 \\
PM-1S4 & 3 & 60 & 5 & 50 & 0.3 & 2.16 & -28.2 & 85.1 & 2.610 \\
PM-1S5 & 5 & 60 & 5 & 50 & 0.3 & 2.35 & -28.6 & 85.7 & 2.671 \\
PM-1S6 & 5.5 & 60 & 5 & 50 & 0.3 & 2.41 & -27.5 & 84.6 & 2.464 \\
PM-1S7 & 0 & 15 & 5 & 50 & 0.3 & 1.49 & -21.2 & 78.5 & 2.130 \\
PM-1S8 & 0 & 30 & 5 & 50 & 0.3 & 1.68 & -26.0 & 81.5 & 2.231 \\
PM-1S9 & 0 & 45 & 5 & 50 & 0.3 & 1.74 & -27.2 & 81.8 & 2.248 \\
PM-1S10 & 0 & 75 & 5 & 50 & 0.3 & 1.89 & -28.3 & 85.1 & 2.611 \\
PM-1S11 & 0 & 90 & 5 & 50 & 0.3 & 1.97 & -28.4 & 85.0 & 2.593 \\
PM-1S12 & 0 & 75 & 4 & 50 & 0.3 & 1.81 & -26.1 & 78.2 & 2.258 \\
PM-1S13 & 0 & 75 & 7.5 & 50 & 0.3 & 2.01 & -26.2 & 79.1 & 2.2 \\
PM-1S14 & 0 & 75 & 5 & 0 & 0.3 & 1.65 & -25.4 & 75.1 & 2.275 \\
PM-1S15 & 0 & 75 & 5 & 30 & 0.3 & 1.74 & -26.2 & 75.8 & 2.082 \\
PM-1S16 & 0 & 75 & 5 & 50 & 0.2 & 1.64 & -24.5 & 75.0 & 2.097 \\
PM-1S17 & 0 & 75 & 5 & 50 & 0.4 & 1.91 & -28.3 & 84.5 & 2.120
\end{tabular}


交联聚苯乙烯微球的热分解峰值温度超过 $400{ }^{\circ} \mathrm{C}$, 说 明交联聚苯乙烯微球具有较好的热稳定性. 随着交联单 体用量的增加, 交联聚苯乙烯微球的热分解峰值温度逐 渐增加, 说明耐热性提高. 如图 3b 所示, 磺化聚苯乙烯 微球的热失重分为 3 个阶段, $20 \sim 120{ }^{\circ} \mathrm{C}$ 的失重为水等 小分子的脱出；180 400 ${ }^{\circ} \mathrm{C}$ 之间的失重对应磺酸基的 脱去 ${ }^{[20]}$; 在 $400 \sim 600{ }^{\circ} \mathrm{C}$ 之间的失重为交联聚苯乙烯的 热分解. 随着交联单体用量增加, 磺化聚苯乙烯微球中 聚苯乙烯载体的热分解峰值温度逐渐增加, 说明交联单 体用量的增加也提高了磺化聚苯乙烯微球的热稳定性. 该磺化聚苯乙烯微球作为固体酸催化剂, 最高使用温度 接近 $200{ }^{\circ} \mathrm{C}$, 满足生物柴油生产要求.
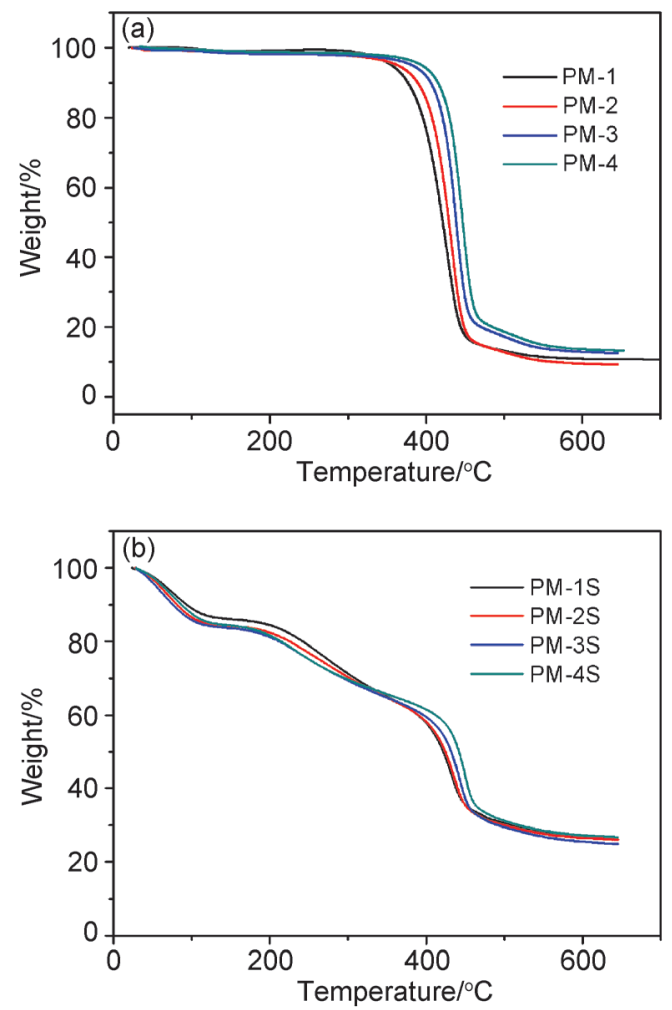

图 3 (a)聚苯乙烯微球和(b)磺化聚苯乙烯微球的热重曲线

Figure 3 TG curves of (a) polystyrene microspheres and (b) sulfonated polystyrene microspheres

表 3 具有不同交联度的磺化聚苯乙烯微球的结构参数

Table 3 Structural parameters of sulfonated polystyrene microspheres with different crosslinking degrees

\begin{tabular}{lcccccc}
\hline Sample $S A_{\mathrm{BET}} /\left(\mathrm{m}^{2} \bullet \mathrm{g}^{-1}\right)$ & $V /\left(\mathrm{cm}^{3} \bullet \mathrm{g}^{-1}\right)$ & $d / \mu \mathrm{m}$ & $\zeta / \mathrm{eV}$ & $D_{\mathrm{S}} / \%$ & {$\left[\mathrm{H}^{+}\right] /\left(\mathrm{mmol} \bullet \mathrm{g}^{-1}\right)$} \\
\hline PM-1S & 162 & 0.44 & 1.89 & -28.3 & 85.1 & 2.611 \\
PM-2S & 181 & 0.43 & 2.02 & -21.2 & 78.6 & 2.097 \\
PM-3S & 214 & 0.39 & 1.96 & -16.3 & 73.2 & 1.861 \\
PM-4S & 240 & 0.38 & 1.99 & -15.1 & 71.1 & 1.653 \\
\hline
\end{tabular}

由表 3 可知, 交联剂用量提高, 磺化聚苯乙烯微球 的比表面积虽然增加, 但是交联度提高, 交联点密度增 加, 孔体积下降. 交联点越密集, 分子链运动越困难, 氯磺酸分子浸入微球内部相对困难, 从而使磺化效率降
低, 磺化度减小, zeta 电位和酸密度也随之下降.

\section{2 磺化聚苯乙烯微球催化制备生物柴油}

以磺化聚苯乙烯微球为酸催化剂(用量为油酸的 $2 \%$, 催化油酸与甲醇酯化反应制备生物柴油; 将其与 含相同酸量的浓硫酸、Ameberlyst-15(简写 A-15)进行对 比，考察其酸催化活性. 由图 4 可见，浓硫酸在反应 $4 \mathrm{~h}$ 时即可达到平衡，转化率达到 $93 \%$, 但是浓硫酸作为酸 催化剂所产生的酸腐蚀、分离回收困难、后处理复杂、 废液较多等问题严重制约了生物柴油的生产 ${ }^{[4,5]}$. 磺化 聚苯乙烯微球催化油酸和甲醇酯化反应 $6 \mathrm{~h}$ 时可达到平 衡, 油酸转化率可达到 $86 \%$. 而 A-15 催化反应 $8 \mathrm{~h}$ 仍未 达到平衡, 油酸转化率也只达到 $37 \%$. 不同酸催化所得 油酸甲酯的产率如图 S1 所示, 也可以看出磺化聚苯乙 烯微球的催化活性稍低于浓硫酸, 但远高于 Ameberlyst-15, 与文献报道的碳基固体酸相当 ${ }^{[16,18]}$. 比 较含有不同交联度的磺化聚苯乙烯微球(PM-1S, PM-2S, PM-3S 和 PM-4S)的催化活性, 可以发现, 微球交联度越 高, 磺化度越低, 酸催化活性亦较低.

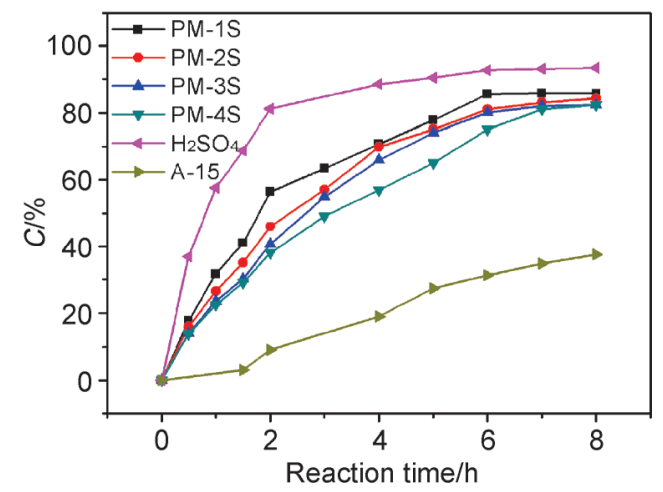

图 4 不同酸催化油酸和甲醇酯化反应转化率-时间曲线

Figure 4 Conversion vs time curves of esterification reaction between oleic acid and methanol catalyzed by different acids

Temperature: $80{ }^{\circ} \mathrm{C}$; oleic acid: $10 \mathrm{~g}$, methanol: $11.34 \mathrm{~g}, \mathrm{H}_{2} \mathrm{SO}_{4}: 0.04 \mathrm{~g}$, the acid amount of other acid catalysts is comparable to that of $\mathrm{H}_{2} \mathrm{SO}_{4}$

催化剂的使用稳定性是衡量催化剂性能的重要指 标，其循环利用更是影响工业化的生产成本. 由图 5 和 图 S2 可见, 回收的磺化聚苯乙烯微球的催化活性基本 维持在较高的水平; 循环使用三次后, 其催化活性可保 持其初始活性的 $92 \%$ 以上. 并且, 循环使用三次后的磺 化聚苯乙烯微球 PM-1SR 的比表面积、粒径、zeta 电位、 磺化度和酸密度与催化前磺化聚苯乙烯微球 PM-1S 相 差无几(表 4); 比表面积和酸密度略有下降, 也反映了 催化活性稍有下降. 此外, 磺化聚苯乙烯微球催化前后 的红外光谱、 $X$ 射线光电子能谱、透射电镜和热重曲线 均非常相似(图 S3 S6), 说明磺化聚苯乙烯微球循环使 用三次后的化学组成、结构形貌和磺化度等均未发生明 显变化. 综上所述, 该磺化聚苯乙烯微球的使用稳定性 好. 


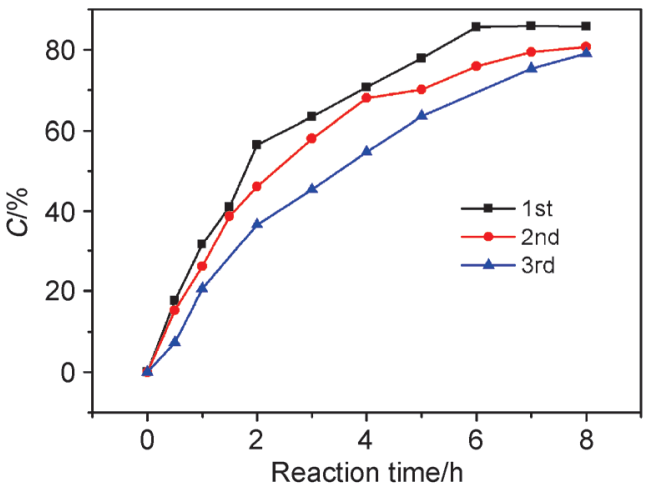

图 5 磺化聚苯乙烯微球循环催化油酸和甲醇酯化反应转化率-时间 曲线

Figure 5 Conversion vs time curves of esterification reaction between oleic acid and methanol catalyzed by sulfonated polystyrene microspheres for several times

Temperature: $80{ }^{\circ} \mathrm{C}$; oleic acid: $10 \mathrm{~g}$, methanol: $11.34 \mathrm{~g}$, catalysis: PM-1S

表 4 磺化聚苯乙烯微球催化前后的性能参数

Table 4 Structure and performance parameters of sulfonated polystyrene microspheres before and after catalysis

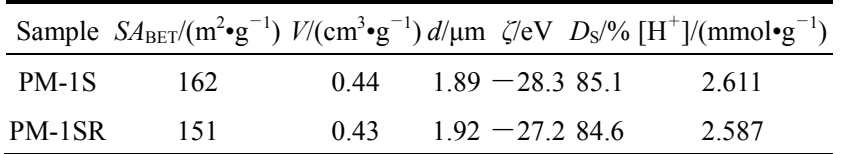

\section{3 结论}

采用了一种简单、绿色和可控方法制备了酸密度可 控、稳定性好的磺化聚苯乙烯微球, 在温和条件下催化 油酸和甲醇酯化制备生物柴油转化率可达到 86\%; 其催 化活性接近于浓硫酸, 远高于离子交换树脂 Ameberlyst-15. 并且, 该磺化聚苯乙烯微球循环使用 3 次后, 其化学组成、结构形貌和磺化度均未发生明显变 化，催化活性仍然可保持其初始活性的 92\%以上.

\section{4 实验部分}

\section{1 交联聚苯乙烯微球的制备}

通过回流-沉淀聚合制备交联聚苯乙烯微球 ${ }^{[14]}$. 在 $100 \mathrm{~mL}$ 圆底烧瓶中加入 $0.45 \mathrm{~g}$ 苯乙烯 $(\mathrm{St}) 、 0.45 \mathrm{~g}$ 二乙 烯基苯(DVB)、0.030 g AIBN 和 $40 \mathrm{~mL}$ 乙腈(ACN), 超 声分散均匀后, 升温至 $90{ }^{\circ} \mathrm{C}$ 恒温进行回流, 反应 $3 \mathrm{~h}$ 后 停止. 将产物以 $6000 \mathrm{r} / \mathrm{min}$ 转速离心 $5 \mathrm{~min}$, 将固体产物 交替加入甲苯和无水乙醇, 超声分散再离心分离, 如此 反复, 每种溶剂分别洗涤三次.

\section{2 磺化聚苯乙烯微球的制备}

参照文献[15]制备磺化聚苯乙烯微球. 在 $100 \mathrm{~mL}$ 的 三口瓶中加入 $0.5 \mathrm{~g}$ 交联聚苯乙烯微球和一定量的溶剂 $\mathrm{CCl}_{4}$, 磁力搅拌溶胀一定时间后, 再加入一定量的氯磺 酸, 在一定温度下磁力摚拌磺化反应一定时间后停止 (配方和工艺条件见表 2). 将产物以 $6000 \mathrm{r} / \mathrm{min}$ 转速离心
$5 \mathrm{~min}$ ，将黄褐色固体产物交替加入乙醇和水，超声分散 再离心分离, 如此反复, 直至洗至中性, 固体产物用水 浸泡过夜. 次日，再分别加入水和乙醇，超声分散再离 心分离, 如此反复, 直至洗至中性.

\section{3 磺化聚苯乙烯微球磺化度和酸密度的测定}

采用热重分析法计算交联聚苯乙烯微球的磺化度 $\left(D_{\mathrm{S}}\right) .180$ 至 $400{ }^{\circ} \mathrm{C}$ 之间的失重主要对应磺酸基的脱除, $400{ }^{\circ} \mathrm{C}$ 时残留物主要为聚苯乙烯. 分别记录 $180{ }^{\circ} \mathrm{C}$ 和 $400{ }^{\circ} \mathrm{C}$ 的热分解残留百分数 $W_{180}$ 和 $W_{400}, M_{\mathrm{SO} H \mathrm{H}}$ 和 $M_{\mathrm{St}}$ 分别是磺酸基和苯乙烯单元的分子量, 按下式计算微球 的磺化度 $D_{\mathrm{S}}$ :

$$
D_{\mathrm{S}}(\%)=\frac{\left(W_{180}-W_{400}\right) / M_{\mathrm{SO}_{3} \mathrm{H}}}{W_{400} / M_{\mathrm{St}}} \times 100 \%
$$

采用酸碱滴定法测定磺化聚苯乙烯微球的酸密度 $\left[\mathrm{H}^{+}\right]\left(\mathrm{mmol} \cdot \mathrm{g}^{-1}\right)$, 具体测定过程如下 ${ }^{[21]}$ : 称取质量为 $m$ (g) 的样品, 放入 $50 \mathrm{~mL}$ 锥形瓶中, 加入 $10 \mathrm{~mL} \mathrm{NaCl}$ (2 $\mathrm{mol} / \mathrm{L}$ ) 溶液, 在室温下搅拌 $24 \mathrm{~h}$, 使微球表面的磺酸基 团 $\left(\mathrm{SO}_{3} \mathrm{H}\right)$ 中的 $\mathrm{H}^{+}$与 $\mathrm{Na}^{+}$充分交换. 离心分离, 取上层清 液, 滴加酚酞做指示剂, 用浓度为 $C_{\mathrm{NaOH}}(\mathrm{mmol} / \mathrm{L})$ 的 $\mathrm{NaOH}$ 标准溶液进行滴定, 使溶液由无色变为红色为止, 记录所用 $\mathrm{NaOH}$ 溶液的体积 $V_{\mathrm{NaOH}}(\mathrm{L})$, 按下式计算微球 的酸密度 $\left[\mathrm{H}^{+}\right]$:

$$
\left[\mathrm{H}^{+}\right]=\frac{C_{\mathrm{NaOH}} \cdot V_{\mathrm{NaOH}}}{m}
$$

\section{4 磺化聚苯乙烯微球催化制备生物柴油}

在带机械搅拌和回流冷凝管的 $100 \mathrm{~mL}$ 三口烧瓶中, 依次加入 $10 \mathrm{~g}$ 油酸、一定量的酸催化剂和 $11.34 \mathrm{~g}$ 甲醇, 超声分散 $5 \mathrm{~min}$, 形成均匀的悬浮液; 在一定转速下 $(350$ $\mathrm{r} / \mathrm{min}$ )室温搅拌 $10 \mathrm{~min}$ 后, 取样并用标准 $\mathrm{KOH}$ 溶液滴 定, 测定初始酸值 $\left(\mathrm{AV}_{0}\right)$. 升温至 $80{ }^{\circ} \mathrm{C}$, 搅拌反应; 间 隔一定时间取样, 测定酸值 $\left(\mathrm{AV}_{x}\right)$, 按下式计算油酸的转 化率 $(C)^{[16]}$.

$$
C(\%)=\frac{\mathrm{AV}_{0}-\mathrm{AV}_{x}}{\mathrm{AV}_{0}} \times 100 \%
$$

反应结束后，静置一定时间，反应产物出现分层， 上层为淡黄色悬浮液, 下层为澄清油状物. 将悬浮液进 行离心分离, 收集固体物, 并用乙醇和丙酮交替洗涤三 次. 真空 $60{ }^{\circ} \mathrm{C}$ 干燥过夜, 再次作为催化剂进行反应.

\section{References}

[1] Pirola, C.; Bianchi, C. L.; Boffito, D. C.; Carvoli, G.; Ragaini, V. Ind. Eng. Chem. Res. 2010, 49, 4601.

[2] Cheng, J.; Qiu, Y.; Huang, R.; Yang, W.; Zhou, J.; Cen, K. Bioresour. Technol. 2016, 221, 344

[3] Shu, Q.; Hou, X. P.; Tang, G. Q.; Liu, F. S.; Yuan, H.; Xu, B. Q.; Zhang, C. X.; Wang, J. F. Chin. J. Inorg. Chem. 2016, 32, 1791. (舒 庆，侯小鹏，唐国强，刘峰生，袁红，许宝泉，张彩霞，王金福， 
无机化学学报, 2016, 32, 1791.)

[4] Liu, F. J.; Huang, K.; Zheng, A. M.; Xiao, F. S.; Dai, S. ACS Catal. 2018, 8, 372 .

[5] Lee, A. F.; Bennett, J. A.; Manayil, J. C.; Wilson, K. Chem. Soc. Rev. 2014, 43, 7887.

[6] De, S.; Dutta, S.; Saha, B. Catal. Sci. Technol. 2016, 6, 7364.

[7] Gupta, P.; Paul, S. Catal. Today 2014, 236, 153.

[8] Lian, Y. F.; Yan, L. L.; Wang, Y.; Qi, X. H. Acta Chim. Sinica 2014, 72, 502. (廉优芬，间碌碌，王羽，漆新华，化学学报，2014，72， 502.)

[9] Akiyama, G.; Matsuda, R.; Sato, H.; Takata, M.; Kitagawa, S. Adv. Mater. 2011, 23, 3294

[10] Wang, Z. Q.; Liu, H. Y.; Cui, H. L.; Zhang, M. H.; Zhang, Z. B. Ind. Eng. Chem. Res. 2015, 54, 7219.

[11] Yu, F.; Smet, M.; Dehaen, W.; Sels, B. F. Chem. Commun. 2016, 52, 2756.

[12] Liu, F. J.; Kong, W. P.; Qi, C. Z.; Zhu, L. F.; Xiao, F. S. ACS Catal. 2012, 2, 565 .
[13] Zhang, X. M.; Zhao, Y. P.; Xu, S. T.; Yang, Y.; Liu, J.; Wei, Y. X.; Yang, Q. H. Nat. Commun. 2014, 5, 3170.

[14] Jin, S.; Pan, Y.; Wang, C. C. Acta Chim. Sinica 2013, 71, 1500. (金 莎, 潘元佳, 汪长春, 化学学报, 2013, 71, 1500.)

[15] Zhou, R.; Wei, R. Q.; Liu, X. N.; Lin, X. J. Chem. Ind. Eng. 2010, 61,1047 . (周莣, 魏荣卿, 刘晓宁, 林弦, 化工学报, 2010, 61, 1047.)

[16] Ma, H.; Li, J. B.; Liu, W. W.; Cheng, B. J.; Cao, X. Y.; Mao, J. D.; Zhu, S. W. J. Agric. Food Chem. 2014, 62, 5345.

[17] Gao, Z. H.; Tang, S. K.; Cui, X. L.; Tian, S. J.; Zhang, M. H. Fuel $\mathbf{2 0 1 5}, 140,669$.

[18] Yu, H. W.; Niu, S. L.; Lu, C. M.; Li, J.; Yang, Y. Z. Fuel 2017, 208, 101.

[19] Yang, Q.; Pan, X. J. ACS Sustainable Chem. Eng. 2016, 4, 4824.

[20] Shu, Q.; Hou, X. P.; Zhu, L. H.; Shen, B. P.; Ma, F.; Wang, J. F. J. Fuel Chem. Technol. 2016, 44, 209. (舒庆, 侯小鹏, 朱丽华, 申邦 坡, 马飞, 王金福, 燃料化学学报, 2016, 44, 209.)

[21] Li, M.; Chen, D. Y.; Zhu, X. F. Chin. J. Catal. 2013, 34, 1674.

(Zhao, C.) 